\title{
Complexity Analysis of Traffic in Corridors-in-the-sky
}

\author{
Min Xue* \\ University of California at Santa Cruz, Moffett Field, CA 94035 \\ Shannon Zelinski ${ }^{\dagger}$ \\ NASA Ames Research Center, Moffett Field, CA 94035
}

\begin{abstract}
The corridors-in-the-sky concept imitates the highway system in ground transportation. The benefit expected from a corridor relies on its capability of handling high density traffic with negligible controller workload, the acceptance of extra fuel or distance, and the complexity reduction in underlying sectors. This work evaluates a selected corridor from these perspectives through simulations. To examine traffic inside the corridor, a corridor traffic simulation tool that can resolve conflicts is developed using $\mathbf{C}$ language. Prescribed conflict resolution maneuvers mimic corridor users' behaviors and conflict resolution counts measure complexity. Different lane options and operational policies are proposed to examine their impacts on complexity. Fuel consumption is calculated and compared for corridor traffic. On the other hand, to investigate the complexity of non-corridor traffic in underlying sectors, the existing Airspace Concept Evaluation System tool is utilized along with the Automated Airspace Concept tool. The number of conflict resolutions is examined and treated as the complexity measurement. The results show heavy traffic can be managed with low complexity for a historical traffic schedule simulated with appropriate operational policies and lane options. For instance, with 608 flights and peak aircraft count of 100, only 84 actions need to be taken in a 24-hour period to resolve the conflicts for an 8-lane corridor. Compared with the fuel consumptions with great circle trajectories, the simulation of corridor traffic shows that the total extra fuel for corridor flights is 26,373 gallons, or $\mathbf{2 . 7 6 \%}$, which is $\mathbf{0 . 3 8 \%}$ less than flying filed flight plans. Without taking climb and descent portions of corridor traffic, the complexity of underlying sectors is reduced by $\mathbf{1 7 . 7 1 \%}$. However the climb and descent portions will eliminate the reduction and the overall complexity of sectors is actually increased by $9.14 \%$.
\end{abstract}

\section{Introduction}

The corridors-in-the-sky ${ }^{1}$ imitate highways in ground transportation by grouping a large number of flights with similar trajectories. The concept is expected to increase overall airspace capacity by allowing high traffic density in corridors with negligible controller workload while reducing the complexity of underlying sectors.

Many studies ${ }^{2-7}$ have shown that a significant number of flights take common trajectories (candidate corridors), and the number increases if the users are willing to travel a little extra distance to join the corridor. In addition, Wing et. al. ${ }^{8}$ conducted an analysis at a conceptual level for a Dynamic Multi-track Airways (DMA) concept, which is similar to the corridor concept. However, the key components on which corridor benefits rely have not been well studied, such as the complexity of handling high density traffic, the extra fuel consumption taken by corridor users, and the complexity of handling non-corridor traffic in underlying sectors. Although Wing et. $\mathrm{al}^{8}$ mentioned the challenge of interactions between DMA (corridor) and non-DMA (non-corridor) traffic, there is no quantitative analysis of the complexity caused by such interactions. Conflict count with no Traffic Flow Management (TFM) actions ${ }^{8}$ and peak aircraft count $^{9}$ have been utilized to assess non-DMA or non-corridor traffic complexity. However, the accuracy of using these metrics to represent complexity is questionable.

\footnotetext{
${ }^{*}$ Research Scientist, University Affiliated Research Center. Mail Stop 210-8. AIAA member

${ }^{\dagger}$ Research Scientist, System Modeling and Optimization Branch. Mail Stop 210-10. email: Shannon.J.Zelinski@nasa.gov, AIAA member
} 
In this work, conflict resolution counts are used to measure complexity. This work first presents a simulation developed for imitating conflict-free operations in corridors. In the simulation, the behaviors of corridor users are approximated by several prescribed conflict resolution maneuvers. Different lane options and policies for resolving conflicts are also explored to find the lowest number of required resolutions. Then corridor complexity is measured and the associated extra fuel consumption is calculated. Additionally, to find the complexity of non-corridor traffic including the complexity caused by the interactions, the Airspace Concept Evaluation System (ACES) ${ }^{10}$ simulation tool with Automated Airspace Concept $(\mathrm{AAC})^{11,12}$ plug-in are applied. Based on a $1 \mathrm{X}$ traffic scenario, the results show that with appropriate operational polices and lane options, the complexity of handling heavy traffic is low. For instance, with 608 flights, only 84 actions need to be taken in a 24-hour period to resolve the conflicts for an 8-lane corridor. The simulation of corridor traffic shows that the total extra fuel for corridor flights is 26,373 gallons, or $2.76 \%$, compared with their great circle trajectories. The simulation of non-corridor traffic shows that the complexity of underlying sectors is increased by $9.14 \%$. The results indicates that the benefit of the corridor is its capability of handling large amount of traffic with relative low complexity and the $2.76 \%$ extra fuel may ba acceptable. However this experiment doesn't show benefit in the complexity of underlying sectors.

The paper will be arranged as follows: Section II describes the corridor model and traffic data. Section III presents the procedures and policies for the simulations of corridor traffic. Section IV shows the results for corridor traffic. After a brief introduction of the ACES AAC simulation, Section V presents the complexity results for non-corridor traffic. Finally, Section VI concludes the paper.

\section{Corridor Model and Traffic Data}

The algorithm from previous work ${ }^{6}$ identified the top corridor candidate. This was a $2 \mathrm{D}$ great circle corridor location such that the number of flights accommodated in the corridor was maximized and the extra flight distance for a flight to join the corridor was less than $5 \%$ of its total flight distance. Based on the historical traffic data taken from Apr.20, 2007, a corridor travelling approximately between San Francisco and New York City via Chicago was identified as the top candidate. A total of 621 flights were identified to be potential users.

Because the above algorithm provides only 2D location, the corridor occupying flight levels must be defined separately. In the study, the corridor occupied three flight levels, FL340, FL350, and FL360. Among them, FL350 served as a passing level. A corridor flight would join the corridor from the passing level and enter another levels when possible. Corridor lane profiles are shown in Fig. 1. Assuming corridor users can perform Required Navigation Performance(RNP) 1.0, which means their lateral deviations from the nominal flight path must stay in \pm RNP or \pm 2.0 nautical miles during $99 \%$ of flight time, each lane had a width of 9 nautical miles. Half of the corridor was for west bound traffic and the other half was for east bound traffic. The number of lanes was a decision variable and was explored in the experiments. The safety zone for individual aircraft was defined as $5 \mathrm{nmi}$ in the horizontal direction and 1,000 $\mathrm{ft}$ in the vertical direction.

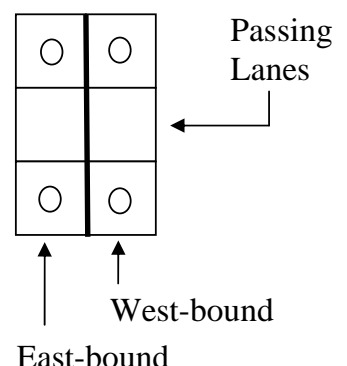

(a)
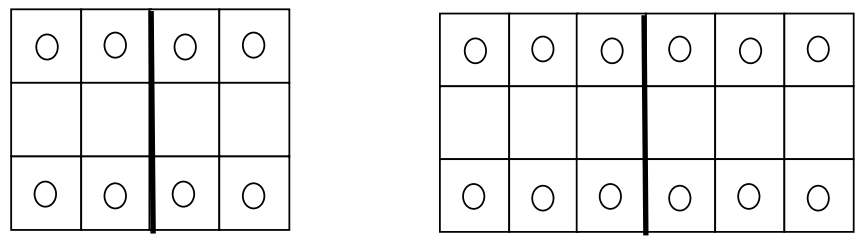

(b) (c)

Figure 1. Lane Profiles (a) 4 lanes (b) 8 lanes (c) 12 lanes

To be practical, aircraft that could not reach the aforementioned levels were ruled out. Furthermore, 
every flight's climb and descent segments were removed. Only the portion between top of climb (TOC) and top of descent (TOD) were considered for corridor operation. The TOC and TOD were computed according to the aircraft's nominal climb and descent rate. Fig. 2 illustrates sample TOC and TOD segments for a flight. Furthermore, if the distance between TOC and TOD was less than $50 \mathrm{nmi}$, the corresponding flight was also ruled out. 608 out of 621 flights satisfied these constraints. 275 flights were east bound and 333 flights were west bound.

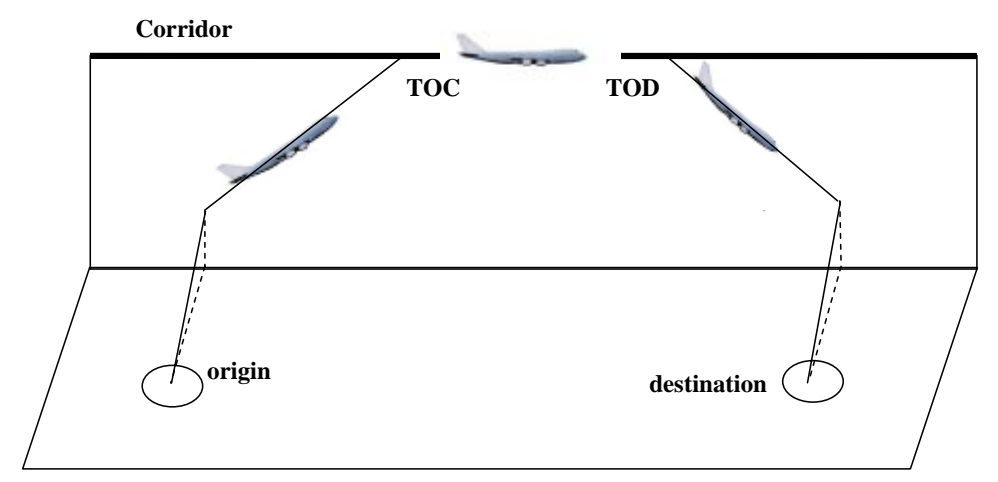

Figure 2. Calculation of TOC and TOD for corridor traffic

Figure 3 shows the time history of aircraft count in the corridor. The peak count for the entire corridor was almost 100 during the given 24 hours. Fig. 4 shows the histogram of flight time for corridor traffic. It can be seen that most of the flights fly in the corridor longer than 50 minutes.

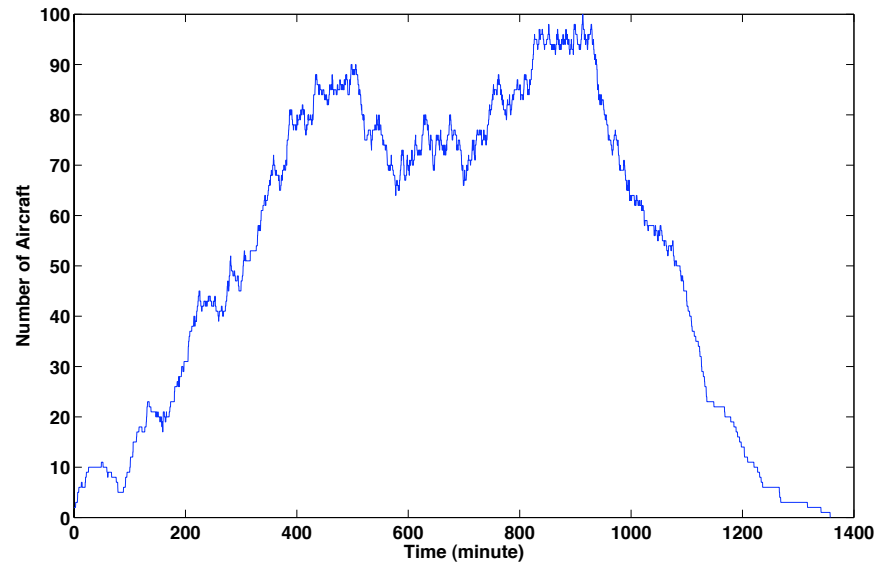

Figure 3. Time History of Aircraft Count

\section{Procedure for Simulating Aircraft Behaviors}

In order to get conflict resolution counts, traffic behaviors are approximated by prescribed maneuvers. Six prescribed maneuvers were applied: climb, descent, left turn, right turn, slowing down, and default cruise maneuver. The left and right turns were defined to be $45^{\circ}$ turns. Climb and descent assumed a nominal rate. The "slowing down" forced the aircraft to fly at the same speed as the flight in front of it such that a conflict would be avoided. The default maneuver was the nominal flight status when there was no conflict or abnormal situation. A typical default maneuver is to fly forward at the cruising speed. All aircraft performances were calculated based on the Base of Aircraft Data (BADA) ${ }^{13}$ developed by Eurocontrol.

To simplify the conflict detection, all maneuvers were descretized into defined time intervals. In this work, the time interval was 0.3 minutes. Each maneuver was divided into several 0.3 minute segments, each lasting 0.3 minutes. Because the duration of a maneuver might not be an exact multiplier of 0.3 minutes, 


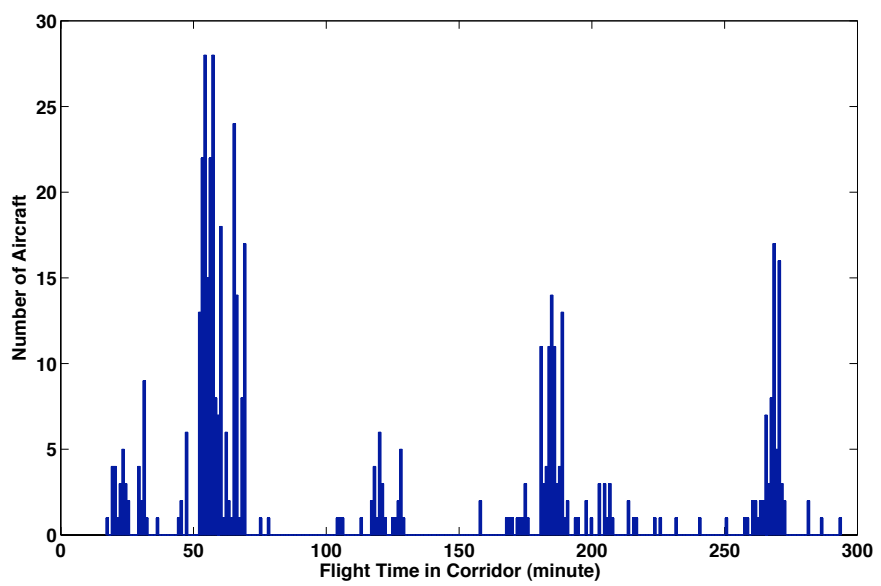

Figure 4. Flight time in the corridor

the last segment (called "tail segment") was extended by adding a "default maneuver" portion such that the duration of the tail segment was 0.3 minutes. Figs. 5(a) and 5(b) demonstrate sample left-turn and climb maneuvers with two regular segments $\left(S_{r}\right)$ and one tail segment $\left(S_{t}\right)$.

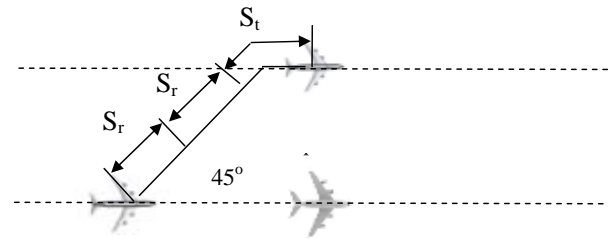

Flow Direction

(a)

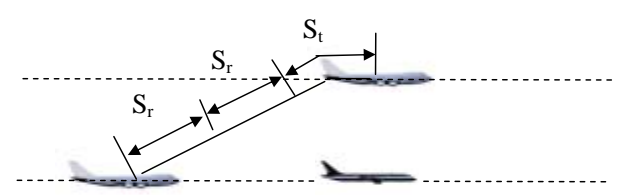

Flow Direction

(b)

Figure 5. Prescribed maneuvers (a) 45 degree left turn (b) climb

Figure 6 presents the work flow of the simulation. Because the durations of prescribed maneuvers normally covered several 0.3 minute steps, a maneuver would not be "conflict-free" unless no conflict occurred during the time steps covering the entire maneuver. As soon as a "conflict-free" maneuver was decided, the postmaneuver position was pre-allocated to prevent any future conflict. Additionally, when a "slowing down" maneuver was chosen, all flights following the aircraft were re-checked for conflicts.

Several viable resolution maneuvers may exist for a conflict depending on operational policies. Two types of operational policies were explored to examine their effects on conflict resolution.

1. "Order-based": the maneuver will be chosen based the following order: default maneuver, left turn, right turn, climb, descent, and slowing down. If the aircraft is at a passing flight level, the order will change to climb, descent, default maneuver, left turn, right turn, and slowing down.

2. "Load-based": instead of a fixed order, the maneuver that balances the lane loads within a given range will be picked. Any maneuver that causes a secondary conflict before the end of the resolution's tail segment is skipped.

Two "load-based" ranges were simulated: "load-based:short" checks lane loads from $10 \mathrm{nmi}$ behind the aircraft to $30 \mathrm{nmi}$ ahead of it, and "load-based:long" checks lane loads form $10 \mathrm{nmi}$ behind to $50 \mathrm{nmi}$ ahead. 


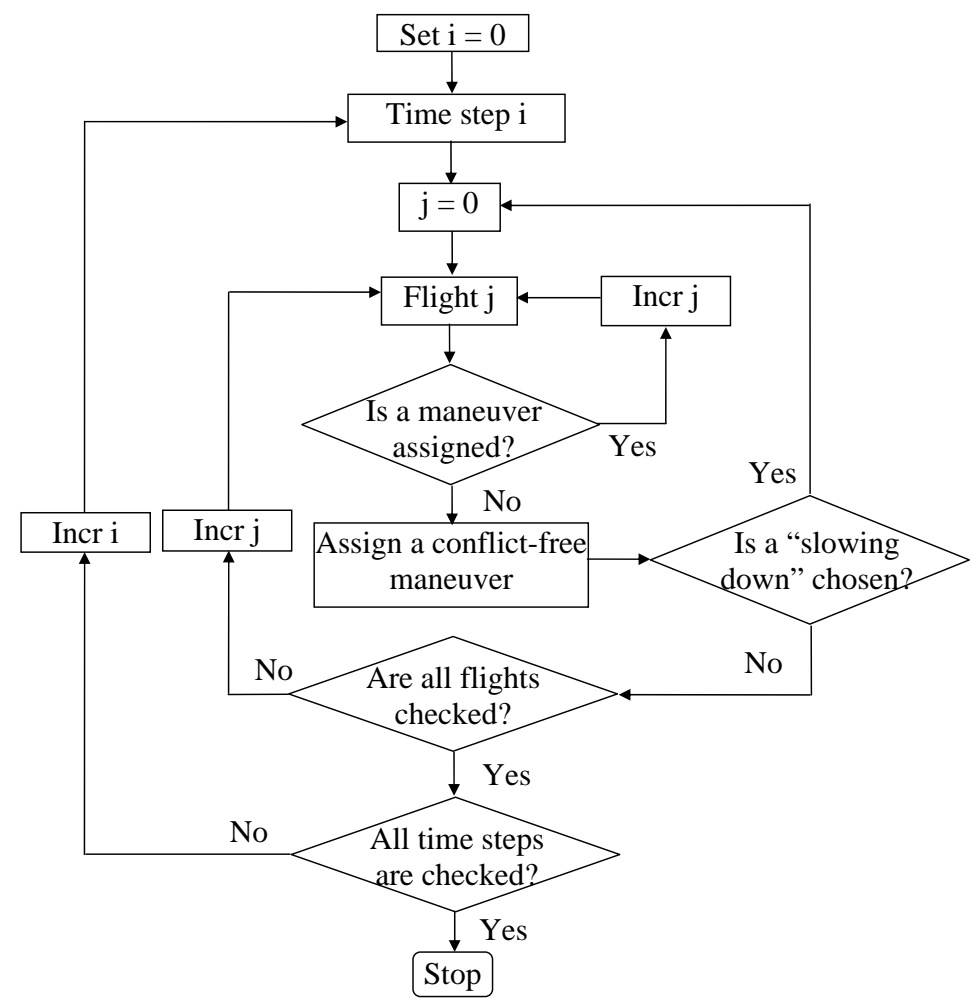

Figure 6. Procedure for Simulating Corridor Traffic

\section{Simulations and Results of Corridor Traffic}

The simulation was implemented in $\mathrm{C}$ language and displayed with Matlab. It was run on a Mac-Pro computer with a dual-core CPU at $2.8 \mathrm{MHz}$. Figure 7 provides a snapshot of an 8 -lane corridor simulation based on the "load-based:long" policy. The horizontal axes are the distances to one end of the corridor (the west end in this case) and the units are nautical miles. For instance, Chicago O'Hare International airport locates at around 2,550 nautical miles away from the west end. The vertical axis in the upper figure is in nautical mile too. The upper figure (Fig. $7(\mathrm{a})$ ) is a top view of 8 lanes (4 lateral, by 2 vertical) of traffic (only 4 can be seen). Among these 4 lanes, the upper 2 lanes are for westbound traffic, and the lower 2 lanes are for eastbound traffic. The lower figure (Fig. 7(b)) presents the side view of the corridor traffic. A red circle or square represents an aircraft in the process of a resolution. A blue circle represents an aircraft during default maneuver.

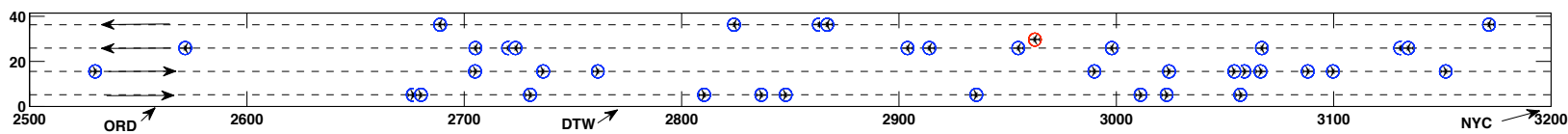

(a)

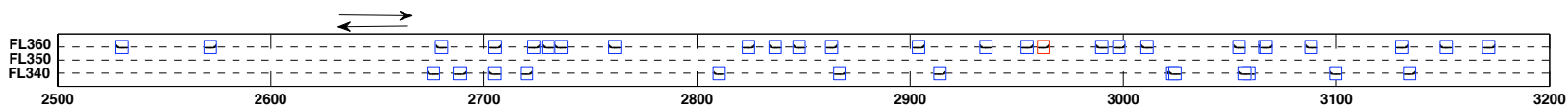

(b)

Figure 7. Snapshot of a simulation (a) top view (b) side view

The experiments explored three operational polices and a various number of lanes. Figure 8 presents the comparison among operation policies and the number of lanes. When there are only two lanes for each 


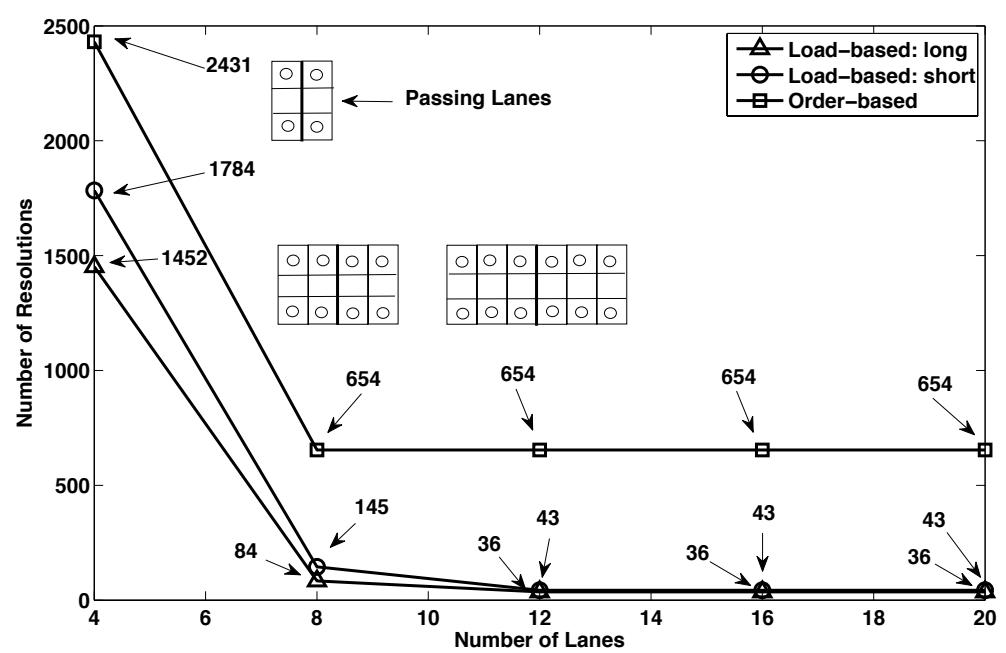

Figure 8. Comparison of Polices and Number of Lanes

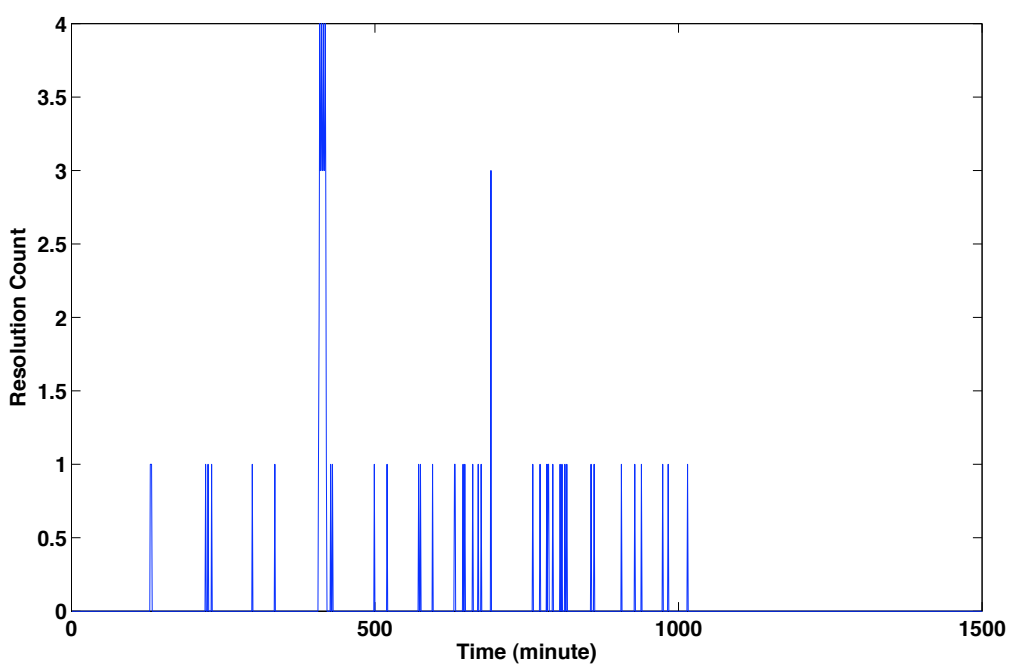

Figure 9. Time History of Resolutions

direction, the number of resolutions for all three policies are high. Because aircraft are only allocated to two lanes, there is little room for improvement using the "load-based" policies. But the load-based policies still have much lower numbers of resolutions than the "order-based" policy. When the number of lanes is doubled to four lanes each way, the resolutions decrease dramatically for all three polices. The "loadbased:long" policy needs 84 resolutions whereas "order-based" requires 654. Obviously, the "load-based" policies outperformed the "order-based" policy. If the number of lanes is 6 in each direction, the resolutions reach minimums at 36 and 43 for "load-based:long" and "load-based:short", respectively. However, the resolution efforts with "order-based" reach a minimum at 654 because the added lanes may not be used at all. These comparisons demonstrate that different rules and different lanes options have a big impact on complexity. On average a sector performs 50 resolutions with peak aircraft less than 20 . Therefore, 84 resolutions is attractive for an airspace with peak aircraft count of 100 . This indicates that handling a large traffic volume traffic in corridors is less complex than in classic sectors. Figure 9 provides the time history of resolutions with the "load-based:long" policy and the 8 lane option, and the peak number of resolutions is only four. The "load-based:long" policy and the 8 lane option will be kept in the following study.

Based on the simulated conflict-free trajectories, the fuel consumption of corridor flights was calculated using BADA3.6. ${ }^{13}$ As a baseline, fuel consumption based on shortest paths (great circle trajectories) and cruise altitudes were calculated. Table 1 lists the number of corridor users and extra fuel consumption 
corresponding to different corridor altitudes. For simplicity, the flight levels shown in the table are the corridor passing lanes. When the corridor altitude is above FL350, many flights are not able to reach the corridor. Whereas when the corridor altitude drops to FL300, although there are 608 aircraft in the corridor, the extra fuel is $8.99 \%$. Because many big jets were forced to fly at altitudes that are far off their optimal cruise altitudes, the extra fuel consumption is high. In the table, the differences between flying the corridor and flying filed flight plans based on historical data are also listed. Flying the FL350 corridor requires less fuel burn than flying filed flight plans. When the altitude of corridor drops to FL300, flying the corridor requires $5.78 \%$ more than flying filed flight plan, which may not be acceptable in operation. The comparisons indicate that the altitude plays an important role in corridor design and analysis, it has to be carefully chosen to maximize the number of corridor users and minimize extra fuel burn. In this experiment, FL350 seems to be the best choice, burning only $2.76 \%$ extra fuel over flying shortest paths.

Table 1. Comparison of Corridors at Different Flight Level

\begin{tabular}{|c|c|c|c|}
\hline Middle flight level & Number of attendees & Extra fuel over great circle & Extra fuel over filed flight plan \\
\hline FL380 & 372 & $0.91 \%$ & $-4.69 \%$ \\
\hline FL370 & 435 & $1.12 \%$ & $-3.56 \%$ \\
\hline FL350 & 608 & $2.76 \%$ & $-0.38 \%$ \\
\hline FL330 & 608 & $4.75 \%$ & $1.66 \%$ \\
\hline FL300 & 608 & $8.99 \%$ & $5.78 \%$ \\
\hline
\end{tabular}

\section{Complexity of Non-corridor Traffic}

The complexity of non-corridor traffic in underlying sectors must be examined to find out the impact of the corridor. Because such large scale human-in-the-loop simulations require lot of resources and resolving conflicts is the most important task for a controller, a preliminary analysis was done by using the number of conflict resolutions as a measure of complexity. The ACES tool was used to simulated the traffic without capacity constraints. In order to perform conflict resolution, the AAC tool was applied as a plug-in of ACES. The AAC simulates the behaviors of resolving conflicts on the basis of predefined maneuvers. The AAC tool used in this work is comprehensive, it can solve three types of problems: conflict resolution, arrival management, and weather avoidance. Because the AAC tool performs 2D maneuvers for conflicts between aircraft and weather polygons and 3D maneuvers for other types of conflicts, modifications were made for this study to allow the tool handle 3D maneuvers for conflicts between aircraft and the designated corridor airspace.

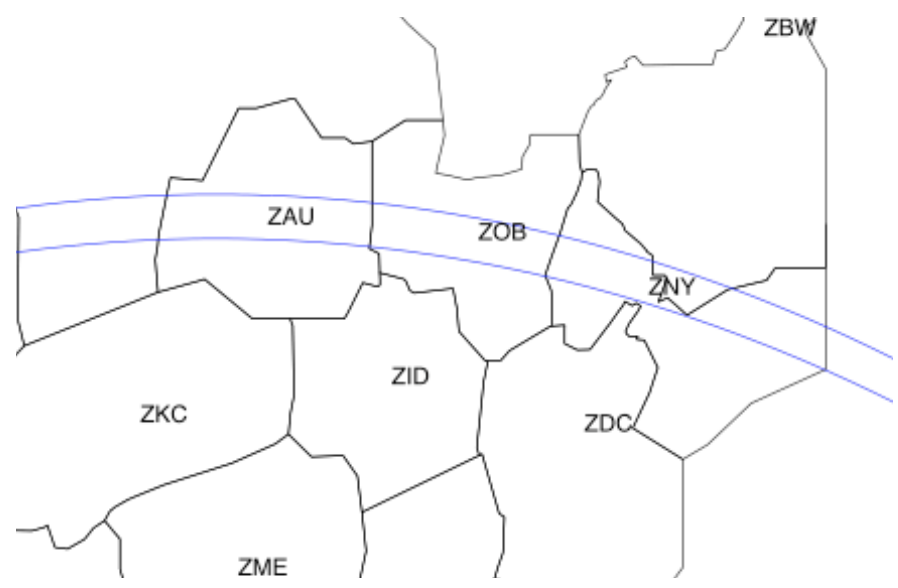

Figure 10. Three Centers and the selected Corridor

Because running the ACES AAC simulation for the entire national airspace is time-consuming, only three 
centers affected by the corridor were chosen and studied. They are Chicago center (ZAU), Cleveland center (ZOB), and New York center (ZNY). Figure 10 shows these three centers and the location and size of the corridor. There were 568 out of 608 corridor flights in these three centers. To get an in-depth analysis, four cases were set up to explore the contribution of complexity. Case I simulated all traffic, including corridor and non-corridor traffic, without any airspace or airport capacity constraints. Case II simulated non-corridor traffic without any capacity constraints and flights flying in the corridor were simply removed. On the basis of Case II, Case III includes the corridor airspace as an obstacle but still no corridor flights. In case III, the corridor was treated as a restricted airspace such that non-corridor traffic need to be capped or tunnelled to avoid the corridor airspace. Finally, Case IV included the climbing and descending portions of corridor traffic. In corridor design the climbing and descending segments of corridor traffic should be taken care of by controllers in surrounding sectors. Among these four cases, Case I represents the current system without corridors. Case IV represents a system surrounding a corridor. In this experiment, the conflict resolutions required in the corridor was excluded.

Table 2. Comparison of complexities

\begin{tabular}{|c|c|c|c|c|}
\hline & Case I & Case II & Case III & Case IV \\
\hline Number of resolutions & 4,856 & 3,978 & 3,996 & 5,300 \\
\hline Difference from Case I & $0 \%$ & $-18.08 \%$ & $-17.71 \%$ & $9.14 \%$ \\
\hline
\end{tabular}

In Table 2 , the complexity in Case II dropped $18.0 \%$ by removing corridor flights, and then it increased a bit in Case III to 3,996 due to the imposed impenetrable corridor airspace. In the experiment, non-corridor flights that would have crossed the corridor were assumed to change their flight plan before departure, so corridor airspace avoidance was not counted as the part of controllers' complexity. However, when climb and descent portions of corridor traffic were involved, in Case IV, the complexity dramatically increased to 5,300. Among the 5,300, 4,070 resolutions happened among non-corridor flights, 855 resolutions were between climb/descent segments and non-corridor flights, and 375 resolutions happened among climb/descent segments. By comparing Case IV and Case I, the complexity increased by $9.14 \%$.

Table 3. Complexity of top 5 busiest sectors

\begin{tabular}{|c|c|c|c|}
\hline Sector & Case I & Case II & Case IV \\
\hline ZNYJF & 342 & $246(-28.0 \%)$ & $346(1.2 \%)$ \\
\hline ZNY75 & 193 & $108(-44.0 \%)$ & $332(72.0 \%)$ \\
\hline ZAU33 & 163 & $140(-14.1 \%)$ & $190(16.6 \%)$ \\
\hline ZOB68 & 116 & $98(-15.5 \%)$ & $110(-5.2 \%)$ \\
\hline ZNY74 & 113 & $85(-24.8 \%)$ & $146(29.2 \%)$ \\
\hline
\end{tabular}

Table 3 provides the complexities of the top 5 busiest sectors in Case I. Sector ZNY75 reduced complexity by $44 \%$ by simply removing corridor flights from the sector, but in taking climb and descent segments of corridor flights into account, the complexity of ZNY75 actually increased by $72.0 \%$. Similar situations happened in other sectors too. Based on these comparisons, the interaction between climb and descent segments of corridor flights and non-corridor flights plays a critical role. It eventually eliminated the benefit of corridors from a complexity perspective. Obviously, in this experiment, the corridor did not show the benefit of reducing complexity.

\section{Conclusion}

This work studied the complexity of traffic in a selected corridor airspace. The corridor's capability of handling high density traffic with negligible controller workload, the acceptance of extra fuel or distance, and complexity reduction in underlying sectors was investigated. A conflict-free traffic simulation is developed for studying the corridor traffic. Prescribed conflict resolution maneuvers mimic corridor users' behaviors and the count of conflict resolutions measures complexity. Different lane options and operational policies 
were proposed to examine their impacts on complexity. Fuel consumption was calculated and compared for corridor traffic. To investigate the complexity of non-corridor traffic in underlying sectors, the ACES tool was used together with AAC to examine the number of conflict resolutions.

With traffic simulated based on historical flight schedule, the results showed that a high traffic volume can be handled with much lower complexity in the corridor airspace than in classic sectors. For instance, with peak aircraft count of 100 , only 84 actions need to be taken in a 24-hour period to resolve the conflicts in an 8-lane corridor. Compared with optimal fuel consumptions with great circle trajectories, the total extra fuel for corridor flights was 26,373 gallons, or $2.76 \%$, which was $0.38 \%$ less than flying filed flight plan. Furthermore, according to the simulations of non-corridor traffic, without taking climb and descent portions of corridor traffic into account, the complexity of underlying sectors was reduced by $17.71 \%$. However, when the climb and descent segments were included, the complexity increased by $9.14 \%$ compared to the operations without the corridor. Although in this experiment with a $1 \mathrm{X}$ traffic scenario, the selected corridor didn't reduce the complexity, further study is needed to determine if the complexity will be reduced when weather is involved or traffic volume reaches $2 \mathrm{X}$ or more.

\section{Acknowledgement}

The authors gratefully acknowledge the contribution of Mr. Chok Fung Lai of University of California at Santa Cruz. Mr. Lai modified the AAC tool and ran all simulations using ACES with AAC plug-in for non-corridor traffic.

\section{References}

\footnotetext{
${ }^{1}$ Kopardekar, P., Bilimoria, K., and Sridhar, B., "Initial Concepts for Dynamic Airspace Configuration," 7th AIAA Aviation Technology, Integration and Operations Conference (ATIO), Belfast, Northern Ireland, 18-20 September 2007.

${ }^{2}$ Alipio, J., Castro, P., Kaing, H., Shahd, N., Sheizai, O., Donohue, G., and Grundmann, K., "Dynamic Airspace Super Sectors (DASS) As High-Density Highways in The Sky for A New US Air Traffic Management System," AIAA/IEEE Digital Avionics Systems Conference, 12-16 October 2003.

${ }^{3}$ Yousefi, A., Donohue, G., and Sherry, L., "High-Volume Tube-Shape Sectors(HTS): A Network of High Capacity Ribbons Connecting Congested City Pairs," Proceedings of the 23rd Digital Avionics Systems Conference, Salt Lake City, CT, 2004.

${ }^{4}$ Sridhar, B., Grabbe, S., Sheth, K., and Bilimoria, K., "Initial Study of Tube Networks for Flexible Airspace Utilization," AIAA Guidance, Navigation, and Control Conference and Exhibit, Keystone, Colorado, 21-24 August 2006.

${ }^{5}$ Gupta, G., Sridhar, B., and Mukherjee, A., "Freeways in the Sky: Exploring Tube Airspace design through Mixed Integer Programming," INFORMS Annual Meeting, Washington, D.C., October 2008.

${ }^{6}$ Xue, M. and Kopardekar, P., "High-Capacity Tube Network Design using the Hough Transform," Journal of Guidance, Control, and Dynamics, Vol. 32, No. 3, 2009, pp. 788-795.

${ }^{7}$ Kotecha, P. and Hwang, I., "Optimization based Tube Network Design for the Next Generation Air Transportation System (NEXTGEN)," AIAA Guidance, Navigation, and Control Conference and Exhibit, Chicago, IL, 10-13 August 2009.

${ }^{8}$ Wing, D. J., Smith, J. C., and Ballin, M. G., "Analysis of a Dynamic Multi-Track Airway Concept for Air Traffic Management," Tech. Rep. NASA/TP-2008-215323, Langley Research Center, Hampton, Virginia, 2008.

${ }^{9}$ Xue, M., "Design Analysis of Corridors-in-the-sky," AIAA Guidance, Navigation, and Control Conference and Exhibit, Chicago, IL, August 10-13 2009.

${ }^{10}$ Meyn, L. and Windhorst, R., "Build 4 of the Airspace Concept Evaluation System," AIAA Modeling and Simulation technologies Conference and Exhibit, Keystone, Colorado, August 12-24 2006.

${ }^{11}$ Erzberger, H., "Transforming the NAS: The Next Generation Air Traffic Control System," the 25th International Congress of the Aeronautical Sciences(ICAS), Yokohama, Japan, August 302004.

${ }^{12}$ Erzberger, H., Lauderdale, T. A., and Cheng, Y., "Automated Conflict Resolution, Arrival Management and Weather Avoidance for ATM," the 27th International Congress of the Aeronautical Sciences(ICAS), Nice, France, September 2010.

${ }^{13}$ Centre, E. E., "BADA Performance Modelling Report," Website, November 2009, http://www.eurocontrol.int/eec/ public/standard_page/ETN_2009_1_BADA.html.
} 